\title{
Thoracic epidural anesthesia for cardiac surgery
}

John P. Williams MD

$\mathrm{T}$

HORACIC epidural anesthesia (TEA) for cardiac surgery has its roots in the groundbreaking work by Blomberg et al. ${ }^{1-4}$ Following this examination of the effect of TEA on patients with coronary artery disease, others including this author were enthused to apply these findings to patients undergoing cardiac surgery. Throughout the 90's, additional work was done to document the role of TEA in cardiac surgery. The major impediment to broader application was then, and is still now, concern regarding epidural hematoma formation.

This refresher course is intended to review both the early and more current findings regarding the benefits of TEA for cardiac surgery as well as review many of the ancillary benefits that accrue from the use of TEA including earlier extubation, improved pain management and improvement in pulmonary function. There will also be a general discussion of the relative risk of epidural hematoma formation when TEA is used during cardiac surgery.

\section{Section A: Myocardial benefits}

Patients with coronary artery disease do not respond to sympathetic activation with the normal coronary dilation. Those areas of the coronary circulation affected by atherosclerosis to any degree demonstrate constriction in response to sympathetic activation. ${ }^{5}$ This is similar to the finding in patients with angina that exercise results in a decrease in diameter of atherosclerotic coronary arteries. ${ }^{6}$ Furthermore, sympathetic activation leads to untoward results in those patients following acute myocardial infarction. ${ }^{7}$ Further, the typical circadian variation in sympathetic activation parallels the risk of arrhythmias, angina and myocardial infarction in patients with coronary artery disease. ${ }^{8,9}$ Finally, previous reports in dogs and rats have documented that, under conditions of experimental coronary occlusion, myocardial infarct size and arrhythmia generation is reduced in the presence of an active thoracic epidural anesthetic. . $^{4,9,10}$

Blomberg et al. documented an increase in stenotic coronary arteries of nearly $64 \%{ }^{2,9}$ Further in a sub- sequent study by this same group, TEA reduced S-T segment depression during exercise stress testing. ${ }^{1,9}$ Further evidence for the ameliorative effect of TEA during coronary occlusion in animals is provided by a study by Tsuchida ${ }^{11}$ where five-minute coronary occlusions led to S-T segment elevation and a concomitant decrease in $\mathrm{pH}$ attenuated by TEA. More recently, investigators from Germany have documented that the normal decrease in wall thickening fraction seen following a ten-minute occlusion of the left anterior descending artery improves much faster in the presence of TEA. ${ }^{9}, 12$ In fact, in this study, wall-thickening fractions returned to $90 \%$ of base line within six hours in the thoracic epidural group while a similar increase did not occur in the control group until 48 hr.

Additionally, TEA is associated with a decrease in both supraventricular and ventricular arrhythmias. ${ }^{13-15}$ In dogs receiving inhalational anesthesia with halothane, the dose of epinephrine required to induce arrhythmia was significantly larger in the presence of TEA. A similar effect was seen in a study with rats in which the incidence of ventricular arrhythmias after coronary ligation was $20 \%$ in the TEA group compared to $53 \%$ in the control group. ${ }^{4}$

Finally, epidural anesthesia is also known to decrease hypercoagulability. ${ }^{16,17}$ Plasminogen activator inhibitor $l$ is decreased in patients who receive epidural anesthesia compared to those who receive general anesthesia. ${ }^{16}$ Others have noted a general decrease in thrombosis following regional $v s$ general anesthesia. ${ }^{16}$ Plasminogen activator inhibitor 1 has been implicated in the genesis of unstable angina and myocardial infarction. ${ }^{9}{ }^{16}$ Thus, there are many reasons to believe that there are myocardial benefits to be garnered from the use of thoracic epidurals during cardiac surgery.

In more recent examinations of cardiac sympathetic activity and myocardial blood flow, Kirno and coworkers demonstrated that cardiac norepinephrine spillover in ten patients receiving TEA was reduced at the time of sternotomy to below that of the pre-surgi-

\footnotetext{
From the Department of Anesthesiology, University of Pittsburgh, Pittsburgh, Pennsylvania, USA.

Address correspondence to: Dr. John P. Williams, Department of Anesthesiology, University of Pittsburgh, A-1305 Scaife Hall, 3550 Terrace Street, Pittsburgh, PA 15261, USA. Phone: 412-648-9624; Fax: 412-648-1887; E-mail: jwilliam@pitt.edu
} 
cal level in control patients $\left(17 \pm 2 \mathrm{ng} \cdot \mathrm{min}^{-1}\right.$ vs $21 \pm 3$ ng. $\left.\min ^{-1}\right) \cdot{ }^{19}$ Furthermore during sternotomy, the control group demonstrated a two and a half-fold increase in norepinephrine spillover. These changes were associated with a $55 \%$ increase in mean arterial pressure mainly related to an increase in systemic vascular resistance in the control group, mean pulmonary artery pressure as well as pulmonary-capillary wedge pressure also increased significantly more in the control group vs the TEA group, $28 v s 9 \%$ and 40 vs $2 \%$, respectively, during surgical stress. The control group received fentanyl in incremental doses of $10-15 \mu \mathrm{g} \cdot \mathrm{kg}^{-1}$ prior to sternotomy and received $70 \%$ nitrous oxide in oxygen following the induction of anesthesia with thiopental $3-5 \mathrm{mg} \cdot \mathrm{kg}^{-1}$. The greater cardiac vein flow and regional myocardial oxygen uptake were significantly lower in the TEA group both before and during sternotomy and TEA attenuated the increase in coronary perfusion pressure, greater coronary vein flow and regional myocardial oxygen uptake during sternotomy. None of the patients in the TEA group had metabolic or electrocardiographic signs of myocardial ischemia while three patients in the control group had indices of myocardial ischemia prior to or during surgery. The major downfall of this study is that it is not representative of what most American anesthesiologists would consider to be a standard anesthetic.

In a more relevant comparison, Stenseth et al. ${ }^{20-22}$ examined the effect of TEA during coronary artery surgery in 30 otherwise healthy, male patients less than $65 \mathrm{yr}$ of age. Three groups were compared, a high dose fentanyl group receiving $55 \mu \mathrm{g} \cdot \mathrm{kg}^{-1}$, a high dose plus TEA group and a low dose fentanyl group receiving 15 $\mu \mathrm{g} \cdot \mathrm{kg}^{-1}$ of fentanyl plus TEA. Almost all patients were receiving beta-blockers and roughly $50 \%$ in each group were receiving calcium channel blockers. The thoracic epidural was calculated to produce a block from Tl to T12, however, due to heavy sedation following premedication, the extent of the epidural block was not recorded in all patients. Vecuronium was used in all patients for muscle relaxation.

In general, findings were consistent across all groups with a few exceptions. Mean pulmonary artery pressure was lower in the high dose fentanyl group than either the high dose fentanyl plus TEA or the low dose fentanyl plus thoracic epidural anesthesia. Pulmonary-capillary wedge pressure was slightly lower in the high dose fentanyl group but not significantly so. Systemic vascular resistance was lower in the low dose fentanyl plus thoracic epidural anesthesia group than the high dose fentanyl group. Eighty-nine percent of epidural group patients needed small doses of ephedrine and more of these patients required methoxamine during extracorporeal circulation. More patients in the high dose fentanyl group required propranolol and nitroglycerine compared with either of the TEA groups.

Similarly, in the postoperative period, nitroprusside was required more commonly in the high dose fentanyl group that in either of the TEA groups. There was very little difference in fluid balance intraoperatively or by one day postoperatively. There was a tendency towards slightly lower postoperative transfusions in the TEA group. Effects on adrenaline, noradrenaline, glucose, cortisol, lactate and free fatty acids were reported in a separate paper. ${ }^{21}$ A significant increase was noted in adrenaline, noradrenaline and systemic vascular resistance in the high dose fentanyl group but this increase was blocked in both of the epidural groups. There was an increase in glucose and cortisol in all groups but the increase was delayed in both of the epidural groups. Thus the use of thoracic epidural anesthesia in a more common setting of low dose fentanyl anesthesia when compared to high dose fentanyl anesthesia with controlled hemodynamics results in improved hemodynamic control and less intervention by the anesthesiologist.

When these results are compared with Kirno's paper it is quite clear that a number of benefits can be attributed to TEA including the reduction in sympathetic overdrive to the myocardium during periods of high stress. Further, TEA offers the advantage of attenuating all measures of the stress response. The one question in this study is whether the height of the epidural blockade was sufficient? The catheter was inserted at $\mathrm{T} 5$ and since the peak heart rates in the low dose fentanyl and TEA groups was 91 at nine hours following the end of extracorporeal circulation, this suggests that in the postoperative period the epidural level was allowed to regress at the cranial terminus.

Other investigators have similarly noted a reduction in the plasma concentration of free fatty acids. This effect was most notable when free fatty acid values were very high. It is conceivable that thoracic epidural may ameliorate rises in myocardial oxygen demand at least in part through this free fatty acid lowering affect.

Other methods of suppressing the sympathetic stress response are available. Previously, investigators have described the use of clonidine to attenuate the stress response prior to and during the perioperative experience. ${ }^{23}$ In a recent comparison of TEA and clonidine, it was noted that TEA but not clonidine combined with general anesthesia for coronary bypass demonstrated a beneficial effect on the perioperative stress response and postoperative myocardial ischemia. ${ }^{24}$ The comparison was undertaken in 21 
patients undergoing coronary artery bypass grafting. General anesthesia was induced with a combination of propofol and sufentanil (1-2 $\mathrm{mg} \cdot \mathrm{kg}^{-1}$ and $\mathrm{l}-2$ $\mu \mathrm{g} \cdot \mathrm{kg}^{-1}$, respectively) and pancuronium was used to facilitate intubation. Anesthesia was maintained with a continuous infusion of sufentanil at $\mathrm{l}-2 \mu \mathrm{g} \cdot \mathrm{kg}^{-1} \cdot \mathrm{hr}^{-1}$ and propofol, l-2 $\mathrm{mg} \cdot \mathrm{kg}^{-1} \cdot \mathrm{hr}^{-1}$. Patients receiving TEA received $8-12 \mathrm{mg}$ of bupivacaine, $0.35 \%$ combined with 16-24 $\mu \mathrm{g}$ of sufentanil in order to achieve a spread of T7 to C6. Half of the initial bupivacainesufentanil bolus was repeated two to three hours after initiation. In the clonidine group, patients received the same anesthetic, however, instead of TEA, patients received a clonidine bolus of $4 \mu \mathrm{g} \cdot \mathrm{kg}^{-1}$ iv over $20 \mathrm{~min}$ followed by a $1 \mu \mathrm{g} \cdot \mathrm{kg}^{-1} \cdot \mathrm{min}^{-1}$ infusion until admission to the intensive care unit (ICU). This was then decreased to a $0.2-0.5 \mu \mathrm{g} \cdot \mathrm{kg}^{-1} \cdot \mathrm{min}^{-1}$ infusion for the duration of the study.

All patients experienced an increase in heart rate following termination of extracorporeal circulation, which was related in part to the use of dopamine in most patients to wean from ECC (extracorporeal circulation). However, the heart rate in both the TEA and clonidine groups was significantly lower than in the control group. Post-operatively, norepinephrine levels were increased in all groups, however, the TEA and clonidine groups had less pronounced rises. Similarly, the serum concentrations of troponin increased in all groups $24 \mathrm{hr}$ after ICU admission. New S-T segment elevations of greater than $0.2 \mathrm{mv}$ or $S$-T depressions of greater than or equal to $1.0 \mathrm{mv}$ occurred in greater than $70 \%$ of control patients but in only $40 \%$ of the clonidine and $50 \%$ of the TEA groups.

Despite all the positive findings noted thus far with TEAs, it is important to note that there can be problems related to the use of this technique. Mergner and colleagues reported on a swine model of severe coronary artery stenosis and the use of thoracic epidural analgesia and general anesthesia. ${ }^{25}$ Unfortunately in this model, they induced significant hypotension as well as inducing an almost total sympathectomy, which is quite different than the type of thoracic epidural anesthesia typically used for cardiac surgery. In this model, however, in the area distal to their stenosis, wall thickening declined $31 \%$ from control and $\mathrm{Dp} / \mathrm{Dt}$ max decreased 29.2\%. Interestingly, left ventricular end-diastolic pressure and wall thickening in the normal myocardium did not change significantly. It is also of interest that, in four animals, heart rate changed less than $10 \%$ and that overall heart rate did change significantly. This, again, suggests there was insufficient cardiac blockade but overly aggressive sympathetic blockade to the remainder of the body to result in significant hypotension. Thus, it is important that one remember that reductions in left ventricular wall tension secondary to thoracic epidurals will improve ventricular function. However, inadvertent hypotension in the presence of severe coronary stenoses where complete sympathetic blockade to the myocardium is not achieved is likely to result in worsening or aggravation of myocardial ischemia.

Finally, studies in awake dogs either with or without autonomic nervous system blockade have examined the role of the cardiac monophasic action potentials and effective refractoriness. ${ }^{13}$ In this study, seven mongrel dogs were anesthetized and 18 gauge catheters were subsequently inserted into the descending aorta in the left atrium for pressure measurement and withdrawal of blood. Following which, a pressure microtransducer was inserted into the left ventricle for left ventricular pressure and rate of increase of left ventricular pressure. All catheters were tunnelled $s c$ and the animals were then trained daily to become accustomed to the experimental environment. Following recovery from the thoracotomy, the dogs were anesthetized and an epidural catheter was inserted percutaneously and then tunnelled to exit near the remainder of the catheters. The correct position of the catheter was then verified radiologically including a spread of local anesthetic combined with contrast solution to determine spread characteristics. After baseline measurements in the awake dog, thoracic epidural anesthesia was induced with the injection of $0.4 \mathrm{mg} \cdot \mathrm{kg}^{-1}$ of lidocaine $2 \%$. Autonomic nervous system blockade was performed by simultaneous injection of $2 \mathrm{mg} \cdot \mathrm{kg}^{-1}$ of propranolol and $3 \mathrm{mg} \cdot \mathrm{kg}^{-1}$ of atropine and $20 \mathrm{mg} \cdot \mathrm{kg}^{-1}$ of hexamethonium. Spontaneous heart rate prior to injection was 118 \pm 15 beats $\mathrm{min}^{-1}$. All measurements of electrocardiographic variables were performed $30 \mathrm{~min}$ after induction of TEA. During sinus rhythm, heart rate decreased by $10 \%$ with no significant change in QRS, P-R and Q$\mathrm{T}$ interval duration. TEA did increase right ventricular monophasic action potential duration over the entire range of steady state. No spontaneous arrhythmias were observed at the atrial or ventricular level. Action potential duration and refractoriness were significantly prolonged at fast heart rates in the presence of TEA when compared to control animals. This prolongation of repolarization is a well-known principle of any arrhythmic agents. One interesting benefit is that as tachyarrhythmias develop the effect of TEA became more prominent and thus its effect may be more relevant with regard to antiarrhythmic potency at higher heart rates.

\section{Section B: Pain control}

Pain control with these thoracic epidurals is probably 
the most easily documented benefit. All studies examining the use of thoracic epidurals for cardiac surgery have documented superior pain relief when compared to all other modalities, including intrathecal opiate analgesia.

In our own study, we examined the course of approximately 60 patients for $48 \mathrm{hr}$ postoperatively. Patients were then divided into four groups - those that received epidural anesthesia alone; those that received epidural anesthesia plus intrathecal analgesia; those that received intrathecal analgesia alone and those that received a standard anesthetic with no neuraxial intervention. The average pain scores for the epidural groups either with or without intrathecal analgesia were not significantly different from one another and both resulted in an average visual analog scale (VAS) pain score (scored from 1 to 10 ) of 0.95 . The intrathecal analgesia alone resulted in a pain score of approximately 2.0 while the control group had a pain score of approximately 3.0 for the entire period of the study. When examined at four-hour intervals, the group receiving the combined intrathecal and epidural analgesia remained less than 1.5 throughout the duration of the study with peak scores occurring between hours 40 and 44. The group receiving epidural anesthesia alone had a peak at approximately 28 to $32 \mathrm{hr}$, however, similarly, peak score remained under 1.5. The intrathecal analgesia group had a continuously increasing pain score peaking at 44 to $48 \mathrm{hr}$ at approximately 2.6. The standard analgesic group reported a peak pain score of 4.0 at approximately hr 32 to 36 , postdeparture from the operating room. As might be expected, the dose of parental medications corresponded well with the peaks in the VAS scores and as expected, morphine utilization in the control group was significantly higher than either of the epidural groups.

\section{Section C: Ancillary benefits}

The benefits of improved pain relief are certainly documented in the previous section by the reduction of the stress response. Additional benefits include earlier extubation which is seen in most studies using thoracic epidurals as well as a lessened incidence of ischemia which may be related either to the decrease in sympathetic activation or an improvement in pain relief, a decrease in the incidence of tachycardia related to pain related changes and a decrease in cardiac output which increases as a consequence of both stress and pain.

Additional ancillary benefits include the decrease in the rate of thrombosis, which is associated with the use of all forms of regional anesthesia. While a direct correlation with a decrease in coronary thrombosis has not been documented, it is quite likely that this ancillary benefit applies to all vessels affected by the inhibition of descending sympathetic influences.

\section{Section D: Technique}

Approach and placement

The typical approach to the epidural space is either paramedian or midline. There is little evidence to suggest that the outcomes are very different when either of these two approaches is used. Each approach has its proponents and detractors. To date there is little evidence to suggest that one technique is superior to another.

There is certainly good theoretical evidence to suggest that the paramedian approach is technically easier and perhaps less problematic in the midthoracic regions (from approximately T5 to T10). In both the lumbar and high thoracic and cervical regions, a more direct approach is available, as the spinous processes are not angulated as deeply and thus allow a midline approach.

The pressured syringe and hanging drop techniques are equal in almost all respects. The hanging drop technique is perhaps more sensitive but this same sensitivity when used in inexperienced hands can lead to inappropriate catheter placement and failure of the epidural as a result. What is perhaps most important is the presence of an experienced anesthesiologist. Whether this person is observing or actually involved in the physical placement of the epidural, his/her presence is absolutely critical. The inexperienced or junior trainee should not be allowed to place these occasionally difficult and always critical catheters.

\section{Section E: Timing}

Most papers to date have described placing the epidural catheter during the day preceding surgery. Our own experience is to place the epidural catheter on the day of surgery and to allow approximately 30 min to elapse between catheter placement and onset of surgery. As will be described in Section G, it is not our current belief that the risk of epidural hematoma is related to any type of traumatic insertion. Rather, we believe that it is a specific type of traumatic insertion that results in epidural hematoma formation. It does not make logical sense that the trauma to an epidural vein regardless of size can lead to the collection of blood and the formation of a hematoma of sufficient size and pressure to compress and ultimately restrict blood supply to the spinal cord. The mean pressure driving the spinal cord is approximately 40 $\mathrm{mmHg}$ in normal patients. Thus, it would require an epidural pressure of at least 40 to $45 \mathrm{mmHg}$ to compress and ultimately restrict blood flow to the spinal 
cord. It cannot follow that if venous pressure is normal or even high normal that it can subsequently exceed arterial pressure in the epidural space regardless of whether the epidural space pressure is negative or positive and several studies have shown that it is positive. Pressure in the epidural space can only rise until it equals venous pressure at which point venous blood flow will cease.

Thus, the only possibility for problems related to the timing of surgery is if arterial blood returns through either the catheter or the needle. Thus, it is the recommendation of this author and it is our practice to withdraw sufficient blood to analyze a $\mathrm{p}_{2}$. If the $\mathrm{p} \mathrm{O}_{2}$ is close to arterial then the procedure is halted and the patient is followed closely for the subsequent 12 to $14 \mathrm{hr}$. If the $\mathrm{pO}_{2}$ is within the venous range, there is no change in plans and surgery proceeds.

\section{Section F: Alternative placement}

There are alternative forms of catheter placement for both pain relief as well as sympathetic blockade which consist of primarily the paravertebral area. The paravertebral approach has not been extensively documented with regard to cardiac surgery. There are a number of investigators in Canada who are exploring this technique and one hopes that we will see a publication of their efforts in the very near future.

\section{Section G: Hematoma}

The first part of this discussion has occurred in the preceding section under "Timing", that is the rationale for using p02 determinations on blood withdrawn from the epidural space as a surrogate marker for epidural hematoma formation. The logic and rationale have been described and will not be repeated, what will be covered here is a recent investigation at our institution regarding epidural hematomas.

We examined the occurrence of any epidural hematoma in two hospitals of the University of Pittsburgh system, Presbyterian and Shadyside. We searched an electronic health database that has been in place for the past 15 years. The words used for the search were: epidural, hematoma and spinal. Each case was then reviewed to confirm both diagnosis and outcome. Each case was also reviewed and classified according to several criteria including the occurrence of ancillary medical illnesses, hospital location, hematoma location, length of time to diagnosis and outcome.

This process identified 17 cases that were true spinal or epidural hematomas (SEH). The characteristics of these cases were remarkably similar to those described by many review papers examining the risk factors for epidural hematomas during or following epidural anesthesia.

Of the 17 cases of confirmed SEH, there was a male to female ratio of $13 / 4$. Ages ranged from 32 to $86 \mathrm{yr}$. Seven cases resulted from spontaneous bleeding, seven cases followed spinal surgery and three cases were related to spinal fractures. No findings of SEH were related to spinal or epidural anesthesia. Among the seven patients with spontaneous SEH; two were receiving anti-coagulants for deep vein thrombosis and had elevated PT and PTT; one had hemophilia (type B); four had hypertension; three out of seven had chronic renal or liver disease. Among postoperative SEH patients, two of the seven patients were receiving chemotherapy and radiation therapy and one had ongoing hemodialysis for renal failure. Among the three patients with traumatic SEH, two had ankylosing spondylitis. Six patients had a history of alcohol abuse. All of these results are concurrent so patients could have more than one etiology associated with SEH.

This makes the usual suggestions that one avoid certain patients with certain illnesses somewhat suspect. Certainly it is true that these patients are more likely to suffer spontaneous SEH; however, the occurrence of an epidural hematoma following an epidural anesthetic should not lead to the automatic assumption that the epidural anesthetic is the culprit. There must be much greater evidence that these patients are not just subsets of the larger risk population with NO relationship to the epidural anesthestic at all.

\section{Summary}

Clearly, there is increasing evidence that TEA is a preferred method of adjunctive anesthesia for cardiac surgery. We have previously published the use of this technique for off pump coronary artery bypass surgery either awake or under general anesthesia. What remains to be seen is if paravertebral blocks can provide similar results with no associated risk of neuraxial hematoma formation. Nonetheless, the risk of hematoma formation appears to be so small that it, alone, should never be the determining factor for not selecting TEA for cardiac surgery.

\section{References}

1 Kock M, Blomberg S, Emanuelsson H, Lomsky M, Stromblad SO, Ricksten SE. Thoracic epidural anesthesia improves global and regional left ventricular function during stress-induced myocardial ischemia in patients with coronary artery disease. Anesth Analg 1990; 71: 625-30.

2 Blomberg S, Emanuelsson H, Kvist H, et al. Effects of thoracic epidural anesthesia on coronary arteries and arterioles in patients with coronary artery disease. 
Anesthesiology 1990; 73: 840-7.

3 Blomberg S, Emanuelsson H, Ricksten SE. Thoracic epidural anesthesia and central hemodynamics in patients with unstable angina pectoris. Anesth Analg 1989; 69: 558-62.

4 Blomberg S, Ricksten SE. Effects of thoracic epidural anaesthesia on central haemodynamics compared to cardiac beta adrenoceptor blockade in conscious rats with acute myocardial infarction. Acta Anaesthesiol Scand 1990; 34: 1-7.

5 Gordon JB, Ganz P, Nabel EG, et al. Atherosclerosis influences the vasomotor response of epicardial coronary arteries to exercise. J Clin Invest $1989 ; 83$ : 1946-52.

6 Gage JE, Hess OM, Murakami T, Ritter M, Grimm J, Krayenbuehl HP. Vasoconstriction of stenotic coronary arteries during dynamic exercise in patients with classic angina pectoris: reversibility by nitroglycerin. Circulation 1986; 73: 865-76.

7 Gottlieb SS, McCarter RJ. Comparative effects of three beta blockers (atenolol, metoprolol, and propranolol) on survial after acute myocardial infarction. Am J Cardiol 2001; 87: 823-6.

8 Rocco MB, Nabel EG, Serwyn AP. Circadian rhythms and coronary artery disease. Am J Cardiol 1987; 59: 13C-7C.

9 Meissner A, Rolf N, Van Aken H. Thoracic epidural anesthesia and the patient with heart disease: benefits, risks, and controversies. Anesth Analg 1997; 85: 517-28.

10 Davis RF, DeBoer LW, Maroko PR. Thoracic epidural anesthesia reduces myocardial infarct size after coronary artery occlusion in dogs. Anesth Analg 19861; 65: 711-7.

11 Tsuchida H, Omote T, Miyamoto M, Namiki A, Ichibara $K$, Abiko $\Upsilon$. Effects of thoracic epidural anesthesia on myocardial $\mathrm{pH}$ and metabolism during ischemia. Acta Anaesthesiol Scand 1991; 35: 508-12.

12 Rolf N, Van de Velde M, Wouters PF, Mollhoff T, Weber TP, Van Aken HK. Thoracic epidural anesthesia improves functional recovery from myocardial stunning in conscious dogs. Anesth Analg 1996; 83: 935-40.

13 Meissner A, Eckardt L, Kirchhof P, et al. Effects of thoracic epidural anesthesia with and without autonomic nervous system blockade on cardiac monophasic action potentials and effective refractoriness in awake dogs. Anesthesiology 2001; 95: 132-8; discussion 6A.

14 Sato K, Yamamura T, Murakami F, Yokota $S$, Kemmotsu $O$. Thoracic epidural anaesthesia combined with enflurane anaesthesia reduces atrioventricular conduction in dogs. Can J Anaesth 1990; 37: 813-8.

15 Hotvedt R, Platou ES, Refsum H. Electrophysiological effects of thoracic epidural analgesia in the dog heart in situ. Cardiovasc Res 1983; 17: 259-66.

16 Rosenfeld BA, Beattie C, Christopherson R, et al. The effects of different anesthetic regimens on frbrinolysis and the development of postoperative arterial thrombosis. Perioperative Ischemia Randomized Anesthesia Trial Study Group. Anesthesiology 1993; 79: 435-43.

17 Steele SM, Slaughter TF, Greenberg CS, Reves JG. Epidural anesthesia and analgesia: implications for perioperative coagulability. Anesth Analg 1991; 73: 683-5.

18 Tuman KJ, McCarthy RJ, March RJ, DeLaria GA, Patel RV, Ivankovich AD. Effects of epidural anesthesia and analgesia on coagulation and outcome after major vascular surgery Anesth Analg 1991; 73: 696-704.

19 Kirno K, Friberg P, Grzegorczyk A, Milocco I, Ricksten $S E$, Lundin $S$. Thoracic epidural anesthesia during coronary artery bypass syrgery: effects on cardiac sympathetic activity, myocarcial blood flow and metabolism, and central hemodynamics. Anesth Analg 1994; 79: 1075-81.

20 Stenseth R, Bjella L, Berg EM, Christensen O, Levang $O W$, Gisvold SE. Thoracic epidural analgesia in aortocoronary bypass surgery. I: haemodynamic effects. Acta Anaesthesiol Scand 1994; 38: 826-33.

21 Stenseth R, Bjella L, Berg EM, Christensen O, Levang $O W$, Gisvold SE. Thoracic epidural analgesia in aortocoronary bypass surgery. II: effects on the endocrine metabolic response. Acta Anaesthesiol Scand 1994; 38: 834-9.

22 Stenseth R, Berg EM, Bjella L, Christensen O, Levang $O W$, Gisvold SE. Effects of thoracic epidural analgesia on coronary hemodynamics and myocardial metabolism in coronary artery bypass surgery. J Cardiothorac Vasc Anesth 1995; 9: 503-9.

23 Flacke JW, Flacke WE. Clonidine prevention of myocardial ischemia during cardiac surgery: will this change outcome? J Cardiothoracic Vasc Anesth 1993; 7 : 383-5.

24 Loick HM, Schmidt C, Van Aken H, et al. High thoracic epidural anesthesia, but not clonidine, attenuates the perioperative stress response via sympatholysis and reduces the release of troponin $\mathrm{T}$ in patients undergoing coronary artery bypass grafting. Anesth Analg 1999; 88: 701-9.

25 Mergner GW, Stolte AL, Frame WB, Lim HJ. Combined epidural analgesia and general anesthesia induce ischemia distal to a severe coronary artery stenosis in swine. Anesth Analg 1994; 78: 37-45. 